\title{
Твердые растворы AlGalnSbAs, выращенные на подложках InAs методом зонной перекристаллизации градиентом температуры
}

\author{
(С) Л.С. Лунин ${ }^{1}$, М.Л. Лунина ${ }^{1}$, Д.Л. Алфримова ${ }^{1}$, А.С. Пащенко ${ }^{1}$, О.С. Пащенко ${ }^{1}$, Н.М. Богатов \\ ${ }^{1}$ Федеральный исследовательский центр Южный научный центр Российской академии наук, \\ 344006 Ростов-на-Дону, Россия \\ ${ }^{2}$ Кубанский государственный университет, \\ 350040 Краснодар, Россия \\ E-mail: lunin_Is@mail.ru
}

Поступила в Редакцию 3 марта 2020 г.

В окончательной редакции 10 марта 2020 г.

Принята к публикации 10 марта 2020 г.

Обсуждается выращивание твердых растворов AlGaInSbAs на подложках InAs из жидкой фазы в поле температурного градиента. Проведен расчет параметров и исследованы люминесцентные свойства и спектральные характеристики твердых растворов AlGaInSbAs, изопериодных подложкам InAs. B рамках модели регулярных растворов проведен анализ гетерофазных равновесий в системе $\mathrm{Al}-\mathrm{Ga}-\mathrm{In}-\mathrm{Sb}-\mathrm{As}$. Выявлены области термодинамической устойчивости к спинодальному распаду твердых растворов $\mathrm{AlGaInSbAs}$ и интервалы изопериодичности к подложке InAs.

Ключевые слова: твердые растворы AlGaInSbAs, термодинамические и кристаллохимические параметры, фазовые равновесия, ширина запрещенной зоны, спектральная характеристика, спектр фотолюминесценции.

DOI: 10.21883/FTP.2020.07.49505.9388

\section{1. Введение}

В твердотельной электронике создание новых конструкционных материалов с заданными свойствами и характеристиками зачастую основано на использовании многокомпонентных твердых растворов, что обусловливает актуальность их изучения. К новым перспективным материалам относятся пятикомпонентные твердые растворы (ПТР) соединений $\mathrm{A}^{\mathrm{III}} \mathrm{B}^{\mathrm{V}}$. Интерес к ним вызван возможностью формирования структурно совершенных переходов за счет одновременного согласования параметров решетки $(a)$ и коэффициентов термического расширения (КТР) сопрягаемых материалов [1-4].

Цель данной работы - выращивание эпитаксиальных слоев твердых растворов AlGaInAsSb, изопериодных подложкам InAs, и исследование их свойств.

\section{2. Теоретический анализ}

Расчет периодов кристаллических решеток $a(x, y, z)$, ширины запрещенной зоны $\left(E_{g}\right)$ и условий изопериодического замещения для твердых растворов $\mathrm{Al}_{x} \mathrm{Ga}_{y} \mathrm{In}_{1-x-y} \mathrm{Sb}_{z} \mathrm{As}_{1-z}$ (InAs) проводили по уравнениям [5-8]. Состав изопериодного твердого раствора $\mathrm{Al}_{x} \mathrm{Ga}_{y} \mathrm{In}_{1-x-y} \mathrm{Sb}_{z} \mathrm{As}_{1-z}$ определяли методом линейной интерполяции периодов решеток бинарных компонентов с использованием данных [7]. Ширину запрещенной зоны пятикомпонентного твердого раствора AlGaInSbAs интерполировали по ширине запрещенной зоны двух четырехкомпонентных систем AlGaSbAs и GaInSbAs с замещением компонентов по узлам металлической подрешетки с учетом нелинейного вклада от эффектов смещения.

На рис. 1 представлены расчетные изопериодические линии для $\mathrm{Al}_{x} \mathrm{Ga}_{y} \mathrm{In}_{1-x-y} \mathrm{Sb}_{z} \mathrm{As}_{1-z} / \mathrm{InAs}$.

Общей тенденцией является их смещение в сторону бо́льших $z$ с увеличением концентрации Al в твердом растворе. Изопериодические линии достаточно хорошо

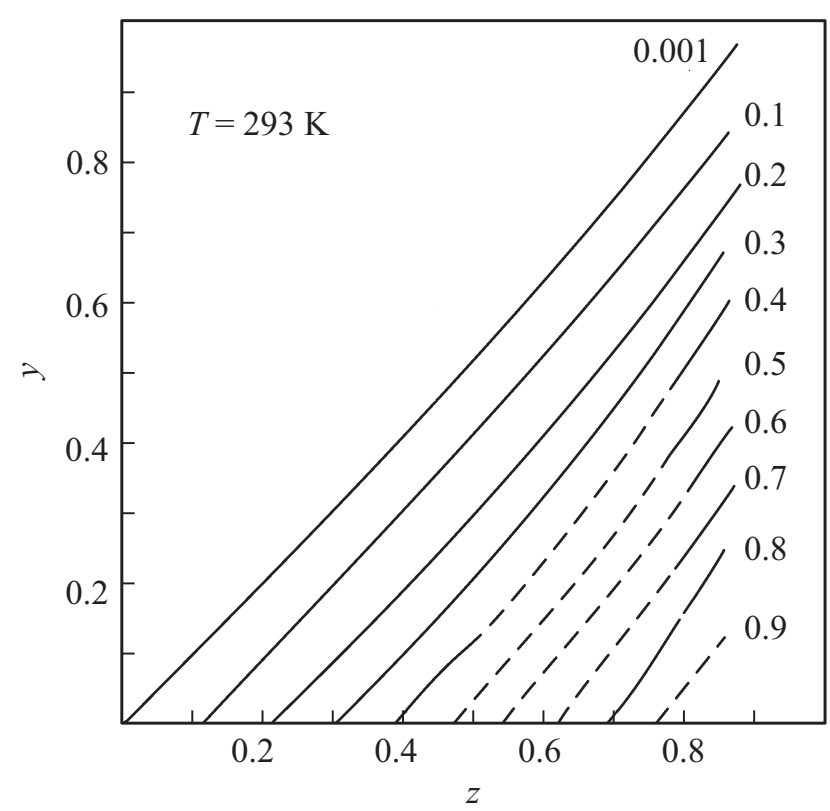

Рис. 1. Концентрационные зависимости $\mathrm{Al}_{x} \mathrm{Ga}_{y} \mathrm{In}_{1-x-y} \mathrm{Sb}_{z} \mathrm{As}_{1-z} / \mathrm{InAs}$ изопериодических линий (пунктиром обозначены области составов, подверженных распаду; цифры у кривых указывают значения содержания $\mathrm{Al}$ в твердой фазе $(x))$. 
описываются уравнением $z=A+B y$ (при $x=$ const). Значения коэффициентов $A$ и $B$ приведены в табл. 1 .

В табл. 2 для фиксированных значений $x$ представлены интервалы измерений параметров $z$ и $y$, в пределах которых твердый раствор, являющийся изопериодическим с подложкой, отличается по КТР на 5 и 20\%.

Таблица 1. Параметры изолиний $z=A+B y$ для пятикомпонентной гетероструктуры $\mathrm{Al}_{x} \mathrm{Ga}_{y} \mathrm{In}_{1-x-y} \mathrm{Sb}_{z} \mathrm{As}_{1-z} / \mathrm{InAs}$ при $x=$ const и $T=293 \mathrm{~K}$

\begin{tabular}{c|c|c|c|c|c}
\hline$x$ & $y_{\min }$ & $y_{\max }$ & $A=z_{\min }$ & $z_{\max }$ & $B$ \\
\hline 0.0 & 0.0 & 1.0 & 0.0000 & 0.9082 & 0.9082 \\
0.1 & 0.0 & 0.9 & 0.0959 & 0.9058 & 0.8998 \\
0.2 & 0.0 & 0.8 & 0.1899 & 0.8985 & 0.8855 \\
0.3 & 0.0 & 0.7 & 0.2839 & 0.8909 & 0.8691 \\
0.4 & 0.0 & 0.6 & 0.3779 & 0.8834 & 0.8425 \\
0.5 & 0.0 & 0.5 & 0.4719 & 0.8760 & 0.8082 \\
0.6 & 0.0 & 0.4 & 0.5475 & 0.8682 & 0.8017 \\
0.7 & 0.0 & 0.3 & 0.6201 & 0.8606 & 0.8008 \\
0.8 & 0.0 & 0.2 & 0.6927 & 0.8529 & 0.8007 \\
0.9 & 0.0 & 0.1 & 0.7653 & 0.8454 & 0.8005
\end{tabular}

Таблица 2. Области составов, где КТР твердого раствора $\mathrm{Al}_{x} \mathrm{Ga}_{y} \mathrm{In}_{1-x-y} \mathrm{Sb}_{z} \mathrm{As}_{1-z}$ и подложки InAs согласуются с точностью 5 и $20 \%$. Значения $y$ определяются из табл. 1 и рис. 1

\begin{tabular}{c|c|c|c|c|c|c|c}
\hline \multirow{2}{*}{$x$} & \multirow{2}{*}{$z_{\min }$} & \multicolumn{2}{|c|}{$z_{\max }$} & \multirow{2}{*}{$x$} & \multicolumn{2}{|c}{$z_{\min }$} \\
\cline { 3 - 4 } \cline { 6 - 7 } & & $\delta \alpha=0.05$ & $\delta \alpha=0.2$ & & & $\delta \alpha=0.05$ & $\delta \alpha=0.2$ \\
\hline 0.0 & 0.00 & 0.15 & 0.65 & 0.5 & 0.45 & 0.65 & 1.00 \\
0.1 & 0.10 & 0.25 & 0.73 & 0.6 & 0.53 & 0.75 & 1.00 \\
0.2 & 0.18 & 0.35 & 0.84 & 0.7 & 0.60 & 0.85 & 1.00 \\
0.3 & 0.28 & 0.44 & 0.93 & 0.8 & 0.68 & 0.92 & 1.00 \\
0.4 & 0.36 & 0.55 & 1.00 & 0.9 & 0.76 & 1.00 & 1.00 \\
- & - & - & - & 1.0 & 0.84 & 1.00 & 1.00
\end{tabular}

Таблица 3. Типы межзонных переходов в пятикомпонентной гетероструктуре $\mathrm{Al}_{x} \mathrm{Ga}_{y} \mathrm{In}_{1-x-y} \mathrm{Sb}_{z} \mathrm{As}_{1-z} / \mathrm{InAs}$

\begin{tabular}{c|c|c|c|c|c}
\hline$x$ & $\begin{array}{c}\text { Интервал } \\
\text { измерения } y\end{array}$ & $\begin{array}{c}\text { Тип } \\
\text { перехода }\end{array}$ & $x$ & $\begin{array}{c}\text { Интервал } \\
\text { измерения } y\end{array}$ & $\begin{array}{c}\text { Тип } \\
\text { перехода }\end{array}$ \\
\hline 0.3 & $0.68-0.70$ & $\mathrm{~L}$ & 0.6 & $0.26-0.40$ & $\mathrm{X}$ \\
0.4 & $0.56-0.60$ & $\mathrm{~L}$ & 0.7 & $0.06-0.30$ & $\mathrm{X}$ \\
0.5 & $0.42-0.46$ & $\mathrm{~L}$ & 0.8 & $0.00-0.20$ & $\mathrm{X}$ \\
0.5 & $0.46-0.50$ & $\mathrm{X}$ & 0.9 & $0.00-0.10$ & $\mathrm{X}$
\end{tabular}

Таблица 4. Области спинодального распада при рассогласовании $\delta \alpha=0.1 \%$ на гетерогранице $\mathrm{Al}_{x} \mathrm{Ga}_{y} \mathrm{In}_{1-x-y} \mathrm{Sb}_{z} \mathrm{As}_{1-z} / \mathrm{InAs}$ $(T=773 \mathrm{~K})$

\begin{tabular}{c|c|c|c|c}
\hline$x$ & $y_{\min }$ & $y_{\max }$ & $z_{\min }$ & $z_{\max }$ \\
\hline 0.4 & 0.1 & 0.42 & 0.47 & 0.74 \\
0.5 & 0.0 & 0.37 & 0.47 & 0.77 \\
0.6 & 0.0 & 0.30 & 0.55 & 0.79 \\
0.7 & 0.0 & 0.15 & 0.62 & 0.74
\end{tabular}

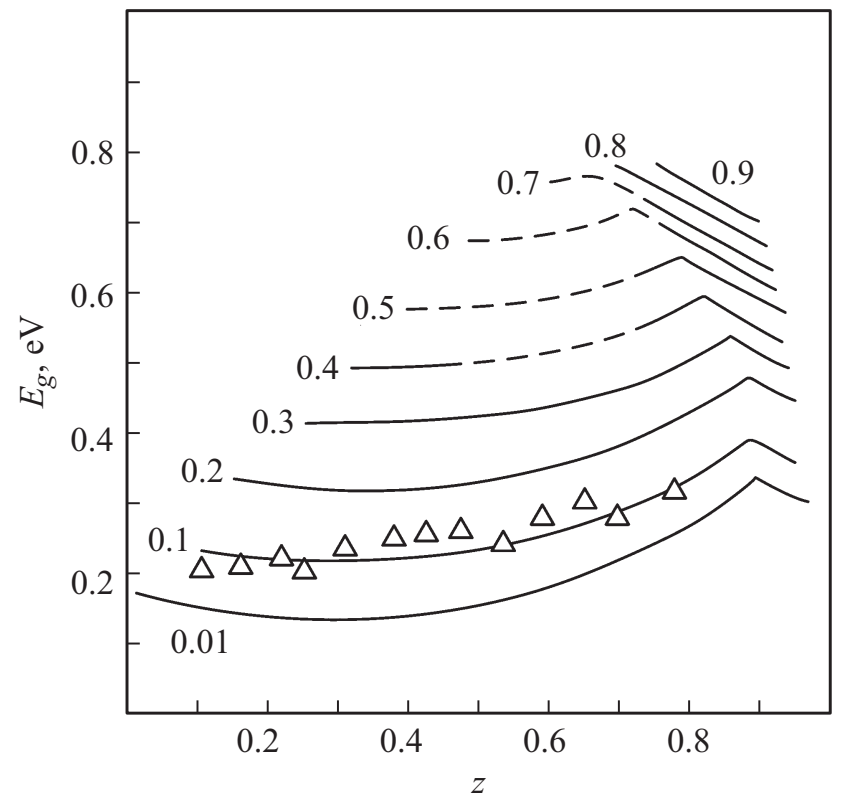

Рис. 2. Концентрационные зависимости $\mathrm{Al}_{x} \mathrm{Ga}_{y} \mathrm{In}_{1-x-y} \mathrm{Sb}_{z} \mathrm{As}_{1-z} / \mathrm{InAs}$ ширины запрещенной зоны (пунктиром обозначены области составов, подверженных распаду; точками обозначены экспериментальные данные; цифры у кривых указывают значения содержания $\mathrm{Al}$ в твердой фазе $(x))$.

Из табл. 2 видно, что интервал значений, в котором КТР слоя и подложки согласуется с точностью до $20 \%$ уменьшается с ростом концентрации алюминия в твердом растворе.

На рис. 2 приведены значения ширины запрещенной зоны $\left(E_{g}\right)$ твердого раствора изопериодического состава.

В твердом растворе AlGaInSbAs существуют прямозонные и непрямозонные переходы в зависимости от состава (табл. 3). Для значений $y$, за пределами указанных в табл. 3, в твердых растворах $\mathrm{Al}_{x} \mathrm{Ga}_{y} \mathrm{In}_{1-x-y} \mathrm{Sb}_{z} \mathrm{As}_{1-z} / \mathrm{InAs}$ наблюдаются прямозонные переходы.

Общая тенденция в изменении ширины запрещенной зоны твердых растворов данного типа заключается в росте $E_{g}$ при повышении концентрации алюминия $x$. При этом с увеличением до определенного значения $z$ при фиксированном х наблюдаются нелинейное возрастание $E_{g}$, а затем линейный спад (рис. 1). С увеличением $x$ точка перелома зависимости $E_{g}(x)$ смещается в сторону меньших значений $z$.

В табл. 4 представлены области спинодального распада при $T=773 \mathrm{~K}$ для твердого раствора AlGaInSbAs без учета возможного влияния напряжений на границе слой-подложка. Видно, что с ростом концентрации алюминия в твердом растворе область спинодального распада расширяется и все большая часть изопериодических составов попадает в зону распада. Однако, как показали расчеты, напряжения на гетерогранице сокращают область спинодального распада (табл. 4). 
Анализ фазовой диаграммы выявляет одну характерную особенность рассматриваемой пятикомпонентной системы. Начиная с $x \geq 0.3$ появляется область, где решения системы уравнений фазового равновесия не существует, так как эта область соответствует области спинодального распада.

\section{3. Экспериментальная часть}

Выращивание эпитаксиальных слоев твердого раствоpa AlGaInSbAs на подложках InAs осуществляли методом зонной перекристаллизации градиентом температуры (ЗПГТ) [9]. К методическим особенностям процесса ЗПГТ относятся: состояние раствора-расплава в момент его контакта с подложкой; толщина слоя растворарасплава, контактирующего с подложкой; кристаллографическая ориентация и качество подготовки поверхности подложки. Для выращивания твердых растворов AlGaInSbAs использовались компоненты, приведенные в табл. 5, также указаны основные операции химической обработки компонентов шихты.

Раствор-расплав формировали из жидкой фазы индия путем насыщения компонентами следующих монокристаллических пластин:

- GaAs (источник галлия и мышьяка) монокристаллический, ориентированный по плоскостям (100) и (111), как нелегированный, так и легированный оловом до концентрации $5 \cdot 10^{17} \mathrm{~cm}^{-3}$;

- InAs (источник мышьяка) монокристаллический (100), как нелегированный, так и легированный серой до концентрации $2 \cdot 10^{18} \mathrm{~cm}^{-3}$.

В зависимости от соотношения существующей концентрации к равновесной при данной температуре растворы-расплавы могут быть ненасыщенными, насыщенными и пересыщенными. Отсутствие равновесия в растворе-расплаве приводит при их изотермическом контакте к растворению подложки InAs. C учетом этих обстоятельств процесс выращивания твердых растворов

Таблица 5. Компоненты шихты для выращивания твердых растворов AlGaInSbAs

\begin{tabular}{c|c}
\hline $\begin{array}{c}\text { Компоненты } \\
\text { шихты }\end{array}$ & $\begin{array}{c}\text { Химическая обработка компонентов } \\
\text { шихты перед процессом эпитаксии }\end{array}$ \\
\hline In 0000 & $\mathrm{HCl}: \mathrm{H} 2 \mathrm{O}(1: 1)$ \\
$\mathrm{Ga} 0000$ & или $\mathrm{HCl}: \mathrm{H}_{2} \mathrm{O}_{2}: \mathrm{H}_{2} \mathrm{O}(1: 1: 0.2)$ \\
$\mathrm{Sb} 000$ & Без обработки \\
$\mathrm{Al} 000$ & $\mathrm{HNO}_{3}: \mathrm{HCl}(3: 1)$ \\
$\begin{array}{c}\mathrm{InAs} \mathrm{(источник} \\
\text { мышьяка) }\end{array}$ & $\mathrm{HCl}_{2} \mathrm{H}_{2} \mathrm{O}(1: 1)$ \\
$\begin{array}{c}\text { GaAs (источник } \\
\text { галлия и мышьяка) }\end{array}$ & $\mathrm{H}_{2} \mathrm{SO}_{4}: \mathrm{H}_{2} \mathrm{O}_{2}: \mathrm{H}_{2} \mathrm{O}(3: 1: 1)$ \\
\end{tabular}

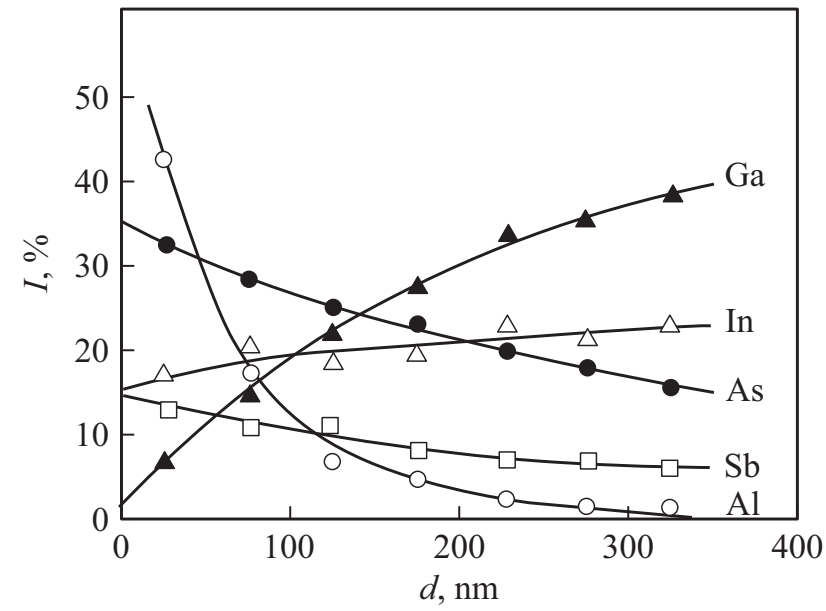

Рис. 3. Профиль распределения компонентов по глубине эпитаксиального слоя твердого раствора AlGaInSbAs/InAs, полученный методом оже-спектроскопии.

AlGaInSbAs необходимо проводить из переохлажденных относительно температуры ликвидуса расплавов на $10 \mathrm{~K}[10]$.

К исходным материалам для проведения эпитаксии из жидкой фазы в поле температурного градиента относится также материал подложки. Мы использовали монокристаллические подложки InAs. Экспериментальные данные показывают, что основные характеристики эпитаксиальных слоев твердых растворов $\mathrm{A}^{\mathrm{III}} \mathrm{B}^{\mathrm{V}}$, полученных методом ЗПГТ, существенно зависят от кристаллографической ориентации и от качества подготовки поверхности [10]. Подготовка пластин предполагает обеспечение планарной зеркальной поверхности с шероховатостью $<10$ нм. При этом поверхность подложки должна быть ориентирована и точно соответствовать заданной кристаллографической плоскости, причем пределы разориентации должны составлять не более $10^{\prime}$. Перед эпитаксией проводились традиционные операции механической шлифовки и полировки исходных пластин InAs c последующими операциями обезжиривания, химической и плазмохимической обработки для удаления нарушенного (аморфизированного) слоя. Для уменьшения шероховатости поверхности подложки InAs до 1 нм применяли ионно-лучевую полировку [11].

Для получения эпитаксиальных слоев твердого раствора AlGaInSbAs постоянного состава использовалась подпитывающая твердая фаза, содержащая компоненты c бо́льшим коэффициентом распределения Al и As (рис. 3).

В качестве подпитывающей твердой фазы применяли предварительно синтезированные поликристаллы AlGaSbAs необходимого состава. Методика синтеза поликристалла описана нами ранее [7].

Толщина жидкой зоны $(l)$ изменялась от 10 до 200 мкм. Сандвичи InAs-In-поликристалл AlGaSbAs помещали в камеру, которую откачивали до $10^{-3}$ Па, а за- 
тем наполняли водородом до 0.5 Па и процесс ЗПГТ проводили в потоке водорода. Эпитаксиальные слои твердых растворов AlGaInSbAs толщиной 2-5 мкм выращивали на подложках InAs при температуре $773 \leq T \leq 973 \mathrm{~K}$ и градиентах $10 \leq T \leq 100 \mathrm{~K} / \mathrm{cm}$. Выбор температурного режима осуществлялся путем исследования температур ликвидуса для каждого отдельного состава с помощью установки визуально-термического анализа in situ по методике [6].

Определение несоответствия параметров решеток подложки и слоя и оценка кристаллического совершенства гетероструктур осуществлялись методом регистрации рентгеновской дифракции. Составы полученных твердых растворов определяли методом масс-спектроскопии вторичных нейтральных частиц. Измерения фотолюминесценции (ФЛ) проводили в спектральном диапазоне от 1500 до 3500 нм при температуре $300 \mathrm{~K}$.

\section{4. Результаты и обсуждение}

На рис. 3 представлены составы эпитаксиальных слоев твердого раствора AlGaInSbAs в виде концентрационных профилей по глубине.

Видно, что содержание всех компонентов по толщине слоев изменяется. Характерной особенностью кристаллизации твердых растворов $\mathrm{AlGaInSbAs}$ на подложке InAs является резкое убывание концентрации $\mathrm{Al}$ и As по толщине слоя, что связано с высокими значениями $K_{\mathrm{Al}}$ и $K_{\mathrm{As}}$ и, следовательно, затрудняет выращивание однородного по составу слоя. Для этого мы и использовали подпитку из поликристалла AlGaSbAs.

Скорость роста эпитаксиальных слоев твердого раствора $\mathrm{A}^{\mathrm{III}} \mathrm{B}^{\mathrm{V}}$ в процессе ЗПГТ зависит от температуры, ее градиента, состава и толщины жидкой зоны. Знание зависимостей скорости роста твердых растворов от толщины жидкой фазы $v(l)$ позволяет выбрать оптимальные условия проведения процесса ЗПГТ. Зависимость $v(l)$ позволяет судить о механизме кристаллизации (или растворения) коэффициентов диффузии в расплаве и скорости испарения летучих компонентов $\mathrm{As}$ и $\mathrm{Sb}$ из жидкой зоны. Для этого необходимо исследовать зависимость $v(l)$ в достаточно широком диапазоне толщин жидких зон, чтобы проявились все три режима (кинетический, диффузионный и смешанный), присущие кристаллизации твердых растворов на подложках соединений $\mathrm{A}^{\mathrm{III}} \mathrm{B}^{\mathrm{V}}$ методом ЗПГТ [9]. Экспериментальные зависимости $v(l, G$ иT $)$ определялись по методике [12] и приведены на рис. 4. На кривых 1 и 2 (рис. 4) четко выражены кинетическая $(0 \leq l \leq 40$ мкм) и диффузионная $(l>60$ мкм) области.

В промежутке между этими областями наблюдается смешанный режим кристаллизации эпитаксиальных слоев AlGaInSbAs на подложках InAs. Как и следовало ожидать, увеличение концентрации алюминия уменьшает скорость кристаллизации эпитаксиального слоя AlGaInSbAs на подложках InAs (кривые 1 и 2) при

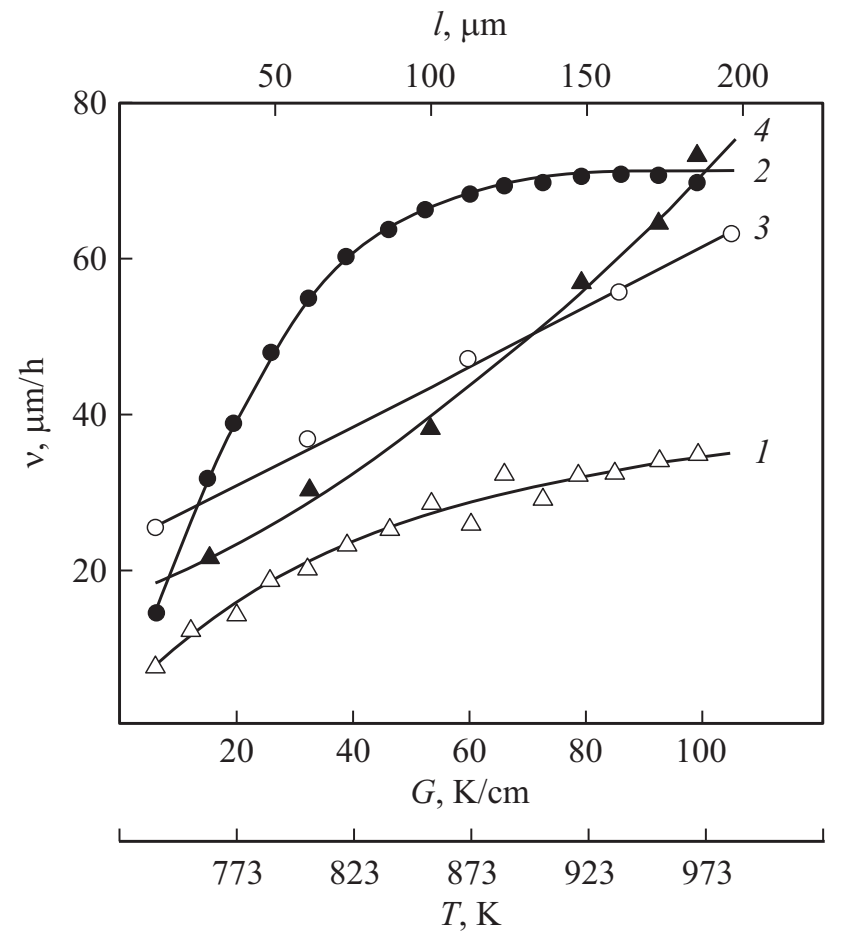

Рис. 4. Зависимости скорости роста $v$ от толщины зоны $v(l)$ (кривые 1,2$)$, от градиента $v(G)$ (кривая 3) и от температуры $v(T)$ (кривая 4) для $\mathrm{Al}_{0.1} \mathrm{Ga}_{0.2} \mathrm{In}_{0.7} \mathrm{Sb}_{0.3} \mathrm{As}_{0.7}$.

всех толщинах зон. Это связано с тем, что увеличение концентрации Al уменьшает коэффициент диффузии в растворе-расплаве $(\mathrm{Al}-\mathrm{Ga}-\mathrm{In}-\mathrm{Sb}-\mathrm{As})$ и замедляет скорость растворения и кристаллизации твердой фазы в расплаве. Наибольшее влияние на скорость кристаллизации (v) слоев AlGaInSbAs на подложках InAs при ЗПГТ оказывает температура процесса $(T)$, чем ее градиент $(G)$ (кривые 3 и 4 на рис. 4).

Спектры ФЛ однородных по составу твердых растворов GaInAs, GaInSbAs и изопериодных $\mathrm{Al}_{0.8} \mathrm{Ga}_{0.1} \mathrm{In}_{0.1} \mathrm{Sb}_{0.82} \mathrm{As}_{0.18}$, выращенных на подложках InAs, полученные при 300 К, приведены на рис. 5.

Так как люминесценция при 300 К в основном обусловлена излучением, связанным с краем зоны, то в спектре ФЛ наблюдается единственный пик. Исследования показали, что с увеличением компонентов твердого раствора увеличивается интенсивность и уменьшается ширина полосы излучения на половине высоты пиков. Интенсивность и ширина полосы излучения определяется в основном кристаллическим совершенством эпитаксиального слоя. В случае трехкомпонентных слоев GaInAs дефектность определяется химическим составом или величиной ширины запрещенной зоны $E_{g}$, поскольку чем сильнее состав слоя отличается от состава подложки, тем больше разность параметров решетки на гетерогранице GaInAs/InAs (рис. 5, кривая 1). В четверных системах наличие дополнительной химической степени свободы позволяет согласовывать параметры 


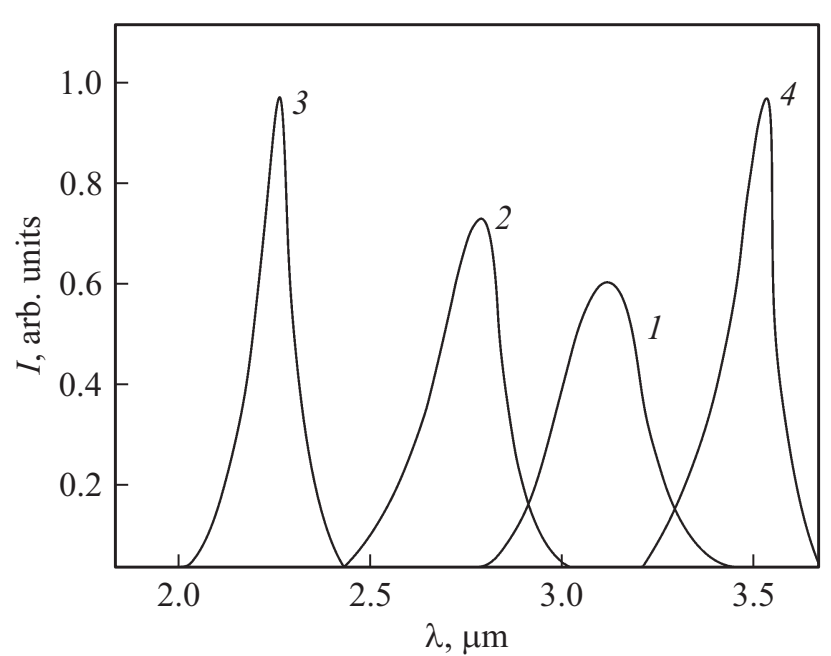

Рис. 5. Спектры фотолюминесценции эпитаксиальных слоев: $1-\mathrm{Ga}_{0.1} \operatorname{In}_{0.9} \mathrm{As}(\mathrm{InAs}), 2-\mathrm{Ga}_{0.6} \operatorname{In}_{0.4} \mathrm{Sb}_{0.4} \mathrm{As}_{0.6}(\mathrm{InAs}), 3-$ $\mathrm{Al}_{0.8} \mathrm{Ga}_{0.1} \mathrm{In}_{0.1} \mathrm{Sb}_{0.7} \mathrm{As}_{0.3}$ (InAs), 4 - InAs (подложка).

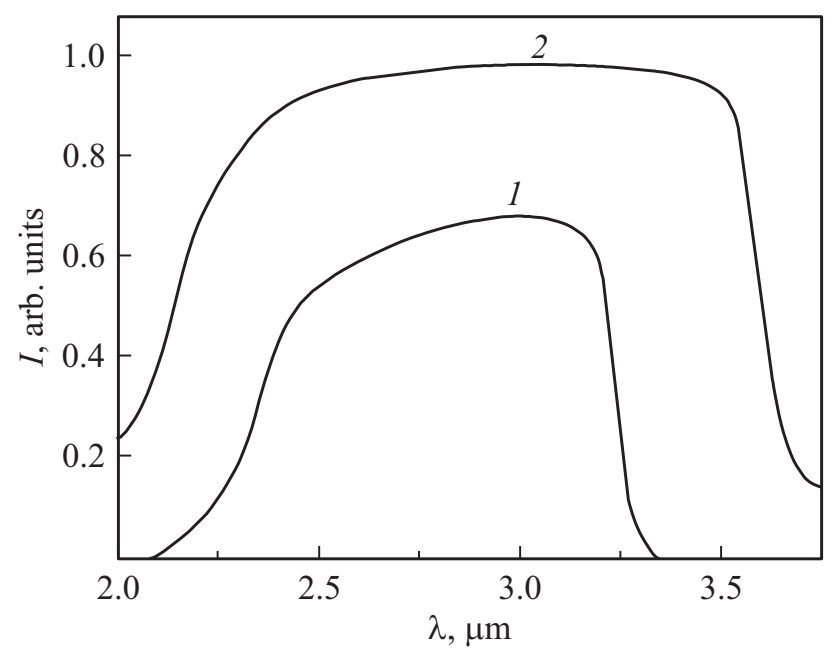

Рис. 6. Внешний квантовый выход гетероструктур: 1 $\mathrm{Ga}_{0.6} \mathrm{In}_{0.4} \mathrm{Sb}_{0.4} \mathrm{As}_{0.6} / \mathrm{InAs,} 2-\mathrm{Al}_{0.35} \mathrm{Ga}_{0.3} \mathrm{In}_{0.35} \mathrm{Sb}_{0.3} \mathrm{As}_{0.7} / \mathrm{InAs}$.

решетки на гетерогранице GaInSbAs/InAs. В этом случае полуширина спектральной полосы ФЛ определяется рассогласованием по КТР слоя GaInSbAs и подложки InAs, причем чем больше разность $\Delta E_{g}$, тем сильнее такое рассогласование и шире полоса излучения (рис. 5, кривая 2). Сравнение спектров ФЛ GaInAs/InAs и GaInSbAs/InAs показывает, что для четырехкомпонентных твердых растворов выше интенсивность, а ширина на половине максимума излучения уменьшается. В пятикомпонентных твердых растворах $\mathrm{AlGaInSbAs(InAs)}$ устраняется рассогласование и по КТР. В результате интенсивность полосы спектра ФЛ увеличивается, а ее ширина на половине максимума излучения уменьшается (рис. 5, кривая 3). Следовательно, с улучшением кристаллического совершенства слоев $\mathrm{AlGaInSbAs}(\mathrm{InAs})$ улучшаются его люминесцентные свойства. Для сравне- ния приведена полоса спектра ФЛ для подложки InAs (кривая 4). Видно, что интенсивность полосы ФЛ и ее ширина для слоя $\mathrm{AlGaInSbAs}$ и подложки InAs сравнимы. Результаты рис. 5 с достаточно высокой степенью согласуются с результатами рис. 1,2 и табл. 4. Положение пика на рис. 5 (кривая 3) соответствует рассчитанной энергии на рис. 2 , для концентраций $x_{\mathrm{Al}}=0.8$ мол. дол. и $z_{\mathrm{Sb}}=0.82$ мол. дол. Кроме того, форма пиков люминесценции подтверждает выдвинутое выше предположение о прямозонных переходах в области термодинамической устойчивости пятикомпонентного твердого раствоpa (табл. 4) $\mathrm{Al}_{x} \mathrm{Ga}_{y} \mathrm{In}_{1-x-y} \mathrm{Sb}_{z} \mathrm{As}_{1-z}$ на подложке InAs.

Спектральные характеристики гетероструктур GaInSbAs/InAs и AlGaInSbAs/InAs приведены на рис. 6 (кривые 1 и 2 соответственно).

Видно, что добавление $\mathrm{Al}$ в качестве пятого компонента в твердый раствор GaInSbAs позволяет значительно повысить внешний квантовый выход до 0.95 и расширить спектральный диапазон $1.5 \leq \lambda \leq 3.5$ мкм.

\section{5. Заключение}

На основе расчетов гетерофазных равновесий в пятикомпонентных системах, основанных на парной аппроксимации квазихимического приближения регулярных растворов, были определены области термодинамической устойчивости твердых растворов AlGaInSbAs, а также интервалы составов и температуры для выращивания изопараметрических слоев твердых растворов AlGaInSbAs на подложках InAs. Исследование кинетики роста эпитаксиальных слоев AlGaInSbAs на подложках InAs показали, что скорость роста зависит от толщины зоны, температуры и ее градиента.

Исследование люминесцентных свойств твердых растворов AlGaInSbAs, выращенных на подложках InAs, показали, что переход к ПТР приводит к увеличению интенсивности и уменьшению ширины полосы излучения на половине высоты пиков, что говорит об их более высоком кристаллическом совершенстве. Исследование спектральных характеристик гетероструктур GaInSbAs/InAs и AlGaInSbAs/InAs показали, что введение пятого компонента $\mathrm{Al}$ увеличивает внешний квантовый выход до $\sim 0.95$ и расширяет спектральный диапазон $1.5 \leq \lambda \leq 3.5$ мкм.

\section{Финансирование работы}

Теоретический анализ и исследование кинетики роста выполнены при финансовой поддержке Российского научного фонда (грант № 19-79-10024), исследования спектральных зависимостей выполнены при финансовой поддержке Российского фонда фундаментальных исследований (проект № 20-08-00108).

\section{Конфликт интересов}

Авторы заявляют, что у них нет конфликта интересов. 


\section{Список литературы}

[1] P.V. Seredin, A.V. Glotov, A.S. Lenshin, I.N. Arsentyev, D.A. Vinokurov, T. Prutskij, H. Leiste, M. Rinke. Semiconductors, 48, 21 (2014).

[2] H.Z. Song, M. Hadi, Y. Zheng, B. Shen, L. Zhang, Z. Ren, R. Gao, Z.M. Wang. Nanoscale Res. Lett., 12 (1), 128 (2017).

[3] S.E. Gulebaglan, E.K. Dogan, M. Aycibin, M.N. Secuk, B. Erdinc, H. Akkus. Cent. Eur. J. Phys., 11, 1680 (2013).

[4] A.G. Gladyshev, I.I. Novikov, L.Ya. Karachinsky, D.V. Denisov, S.A. Blokhin, A.M. Nadtochiy, A.S. Kurochkin, A.Yu. Egorov. Semiconductors, 50, 1186 (2016).

[5] D.L. Alfimova, L.S. Lunin, M.L. Lunina. Inorg. Mater., 50, 113 (2014).

[6] V.V. Kuznetsov, E.A. Kognovitskaya, M.L. Lunina, E.R. Rubtsov. Russ. J. Phys. Chem. A, 85, 2062 (2011).

[7] D.L. Alfimova, L.S. Lunin, M.L. Lunina, A.E. Kazakova, A.S. Pashchenko, S.N. Chebotarev. Inorg. Mater., 53, 1217 (2017).

[8] K. Shim. Phil. Mag., 94, 3088-3097 (2014).

[9] В.Н. Лозовский, Л.С. Лунин, В.П. Попов. Зонная перекристаллизация градиентом температуры полупроводниковых материалов (М., Металлургия, 1987).

[10] В.В. Кузнецов, Л.С. Лунин, В.И. Ратушный. Гетероструктуры на основе четверных и пятерных твердых растворов соединений $\mathrm{A}^{\mathrm{III}} \mathrm{B}^{\mathrm{V}}$ (СКНЦВШ Ростов н/Д., 2003).

[11] С.Н. Чеботарев, А.С. Пащенко, A. Williamson, Л.С. Лунин, В.А. Ирха, В.А. Гамидов. Письма ЖТФ, 41, 13 (2015).

[12] М.Л. Лунина, Л.С. Лунин, В.В. Калинчук, А.Е. Казакова. ФTT, 60, 888 (2018).

Редактор Г.А. Оганесян

\section{AIGalnSbAs solid solutions grown on InAs substrates by zone recrystallization with a temperature gradient}

L.S. Lunin ${ }^{1}$, M.L. Lunina ${ }^{1}$, D.L. Alfimova ${ }^{1}$, A.S. Pashchenko' , O.S. Pashchenko ${ }^{1}$, N.M. Bogatov ${ }^{2}$

${ }^{1}$ Federal Research Centre the Southern Scientific Centre of the Russian Academy of Sciences, 344006 Rostov-on-Don, Russia

${ }^{2}$ Kuban State University, 350040 Krasnodar, Russia

Abstract Growth of AlGaInSbAs solid solutions on InAs substrates from liquid phase in a temperature gradient field is discussed. There are calculated parameters and studied luminescent properties and spectral characteristics of AlGaInSbAs solid solutions isoperiodic to InAs substrates. Within the model of regular solutions, an analysis of heterophased neutrality in $\mathrm{Al}-\mathrm{Ga}-\mathrm{In}-\mathrm{Sb}-\mathrm{As}$ system is carried out. Regions of hermodynamic stability to spinodal decomposition of AlGaInSbAs solid solutions and isoperiodicity intervals to InAs substrate are revealed. 